\title{
Numerical Simulation of Flow Field and Internal Structure Optimization for CFD Based Pipeline Coalescence Device
}

\author{
Na Luo, Jianxiang Jin ${ }^{\text {a) }}$, Heping Ye ${ }^{\text {b) }}$, Chen Xue, Yucheng Zhang, Zhenyu Zhai, \\ Runze Chen, Benzhi Liu ${ }^{c)}$ \\ College of Environmental Science and Engineering, Yancheng Institute of Technology, Yancheng Jiangsu, 224051, \\ China. \\ a)ygjjx2000@163.com \\ b)1770850013@qq.com \\ c)1bzycit@163.com
}

\begin{abstract}
Particles aggregation technology is a kind of effective method to strengthen the collection and removal of ultrafine particles. The flow field of the turbulence coalescence device was simulated by FLUENT, and the results showed that diaphragms in the turbulence coalescence device could produce vortices, which were favorable for the aggregation of the ultrafine particles. Considering the vortices and the pressure drop, "1.5-2.0 times the height of the diaphragm for vertical spacing between two diaphragms" and "2.0-3.0 times the length of the diaphragm for horizontal spacing between two diaphragm groups" are thought of optimal design.
\end{abstract}

Key words: Ultrafine Particle; Coalescence; Flow Field; Numerical Simulation; CFD.

\section{INTRODUCTION}

In view of the low efficiency of the existing collector, it is necessary to study and develop technologies and devices for strengthening ultrafine particles aggregation.

In recent years, many researches and preliminary applications have been carried out in the field of electric coagulation, chemical agglomeration and acoustic agglomeration. As far as the research of coalescence device, Watanabe [1] have designed an improved three zone electric coagulation ESP system based on the principle of electric coagulation, and the related experiments and theoretical studies were carried out at the same time. Zhang gangue et al [2] studied simulation and analysis of collision efficiency in acoustic agglomeration. The results showed that collision efficiency was very low for PM2.5, especially for PM1, which meant that the fine particles were hardly collected by orthogenetic agglomeration. Collision efficiency decreased with acoustic frequency increased, but it enlarged the range of optimum frequency of agglomeration. Han Xiao Liu [3] carried out the study of multiple vortex body's numerical simulation and found that each vortex body had good effect in producing vortex, and the greater flow field spacing, the smaller the highest velocity; the turbulence intensity is increasing gradually from the former vortex body to the next one, and there may be a best spacing between the vortex bodies which makes the best turbulent intensity. Han Gang [4] studied the segment layout optimization on the vortex generating section of superfine powder coagulation device. Based on the designed ultrafine powder coagulation device, CFD software was used to simulate the flow field and particle trajectory on different entrance velocity and different particle sizes of the coagulation device. Entrance to different wind speed, the coagulation device velocity, turbulence intensity, vortices and particle motion trajectory were analyzed, and some experimental data were compared. The best design scheme was obtained by simulation.

In this work, based on fluid mechanics coagulation and bipolar charging technology, a kind of pipeline turbulence coalescence device was invented. The turbulence coalescence device was placed in the flue entrance of 
the electrostatic dust collector, and the effective capture of ultrafine particles can be achieved. A model of the turbulence coalescence device was built and simulated by FLUENT, the flow field of the turbulence coalescence device was studied and the internal structure of the coalescence device was optimized.

\section{PHYSICAL MODEL}

\section{Structure of the Coalescence Device}

The internal structure of the coalescence device is illustrated in Fig.1.

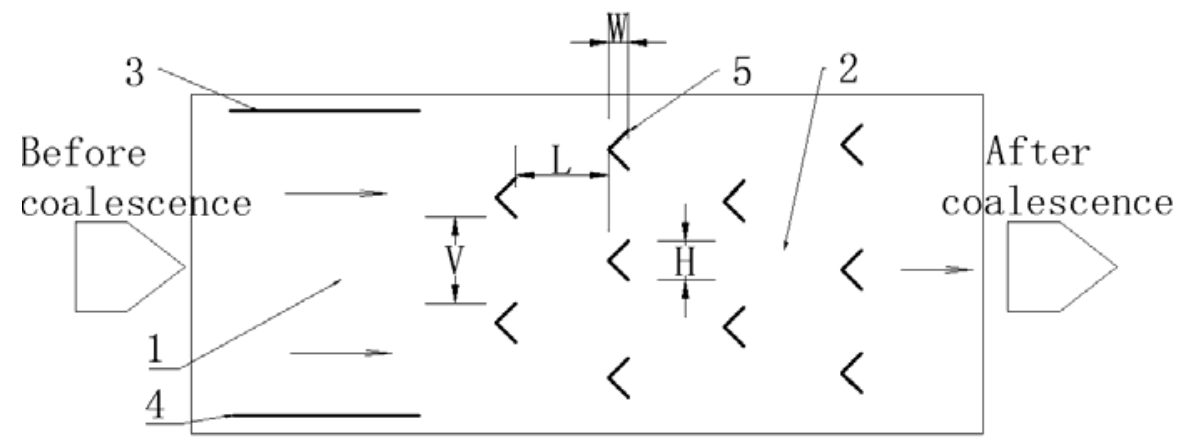

1: charged section, 2: coalescence section, 3: negative plate, 4: positive plate, 5: angle diaphragms,

W: length of angle diaphragms: vertical distance of angle diaphragms,

$\mathrm{H}$ : Height of angle diaphragms: Horizontal distance of angle diaphragms.

FIGURE 1. Structure of pipeline turbulence coalescence device with angle diaphragms.

As shown in Figure 1, the whole turbulence coalescence device is pipe type. The airflow first passes through the charged section and then enters the coalescence section. Positively charged particles and negatively charged particles collide together under the combined action of "electrostatic coagulation" and "turbulent coagulation. 3-4 groups of angle diaphragms, which could create favorable conditions for the aggregation of the ultrafine particle, are set up in coalescence section.

\section{Analysis of the Force of Particles}

The force of the charged particles in the condenser is very complex, including the gravitational force, the Coulomb force between particles, buoyancy, the effect of gas on the drag force, the pressure gradient force, the virtual mass force, the Magnus force, the Coffman force, and the Basset force. The motion equation of charged particles is a two-dimensional horizontal model. Ignoring the gravity effect, according to the analysis of the loading condition of charged particles, the motion equation of charged particles is:

$$
\mathrm{dUp} / \mathrm{dt}=\mathrm{Fs}-\mathrm{Fd}+\mathrm{Fe}
$$

The 3 items on the right are Coffman force, fluid viscous resistance and particle Coulomb force (ignoring secondary force).

\section{Meshing and Boundary Conditions}

Using quadrilateral structured meshing, the mesh quality is controlled to reduce the dependence of the simulation results on the mesh. The mesh around the horizontal diaphragms is encrypted and optimized. The total number of meshes is $38463-38,622$. The inlet boundary conditions adopt the velocity-inlet, and the particle incidence velocity is the same as the gas velocity; the boundary conditions of the outlet adopt fully developed flow conditions; the gas phase wall boundary adopts the no-slip shear condition; the standard wall function is used near the wall surface; the 
gas outlet adopts the escape type, the particle outlet is of the trap type, and the wall and the horizontal strips are of reflect type.

\section{RESULTS AND DISCUSSION}

At the inlet air velocity of $10 \mathrm{~m} / \mathrm{s}$, numerical simulation of the coagulation section of the turbulence coalescence were carried out separately with different angle diaphragms of $\mathrm{V}=1.0 \mathrm{H}, \mathrm{V}=1.5 \mathrm{H}, \mathrm{V}=2.0 \mathrm{H}, \mathrm{V}=3.0 \mathrm{H}$. Fly ash particle size is 2 . $5 \mu \mathrm{m}$. The velocity vectors of flow field in the turbulence coalescence section with different $\mathrm{V}$ values is shown in Fig. 2 .

As can be seen from Fig.2, the turbulence appears around the angle diaphragms. Along the direction of the flow, there are vortices before the diaphragms and there is still a continuation of the vortex effect. When the airflow passes through the turbulence coalescence section, velocity variations are formed surrounding the diaphragms (The length of the arrow represents the velocity value in Fig.2), and there are obvious velocity gradients, which could create favorable conditions for the aggregation of the ultrafine particle. The maximum velocity gradient exists in (a), but the pressure drop is also the largest. The velocity values tend to be uniformly distributed in (c) and (d), especially in (d). It is also shown in Fig. 2 that the velocity direction of ultrafine particles in air flow will change with air flow (instead of advancing along the horizontal direction). The intersection of airflow at different speeds will inevitably bring about the encounter and aggregation of ultrafine particles in airflow, which can significantly enhance the aggregation of the ultrafine particle in the turbulence coalescence section. The effect of changing direction is best in (a). Thus, with the increase of $\mathrm{V}$, the effect of turbulence will be worse, but too small $\mathrm{V}$ value will cause excessive device pressure drop. $\mathrm{V}=1.5-2.0 \mathrm{H}$ is an optimal choice. It will also be used in the following numerical simulation.

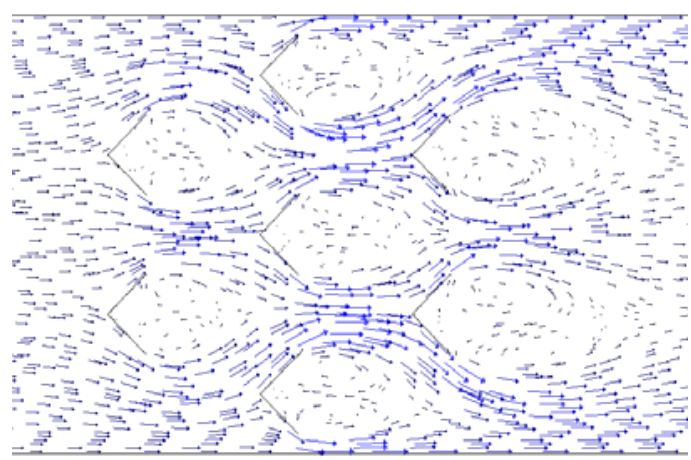

(a)

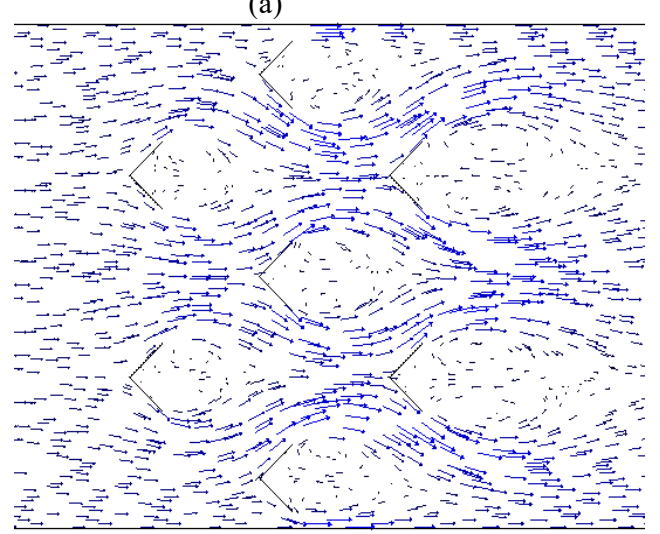

(c)

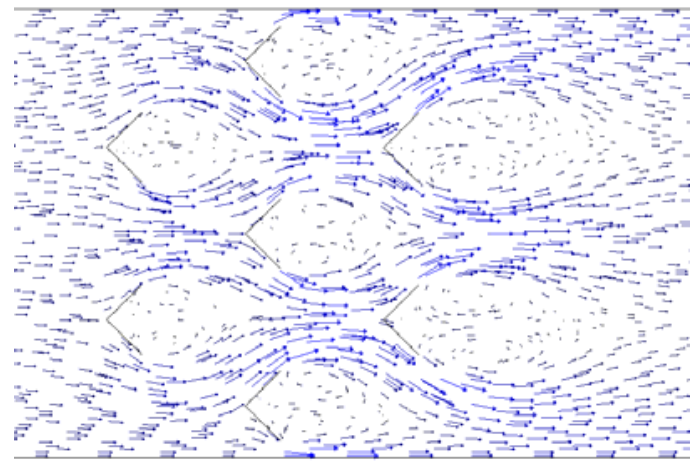

(b)

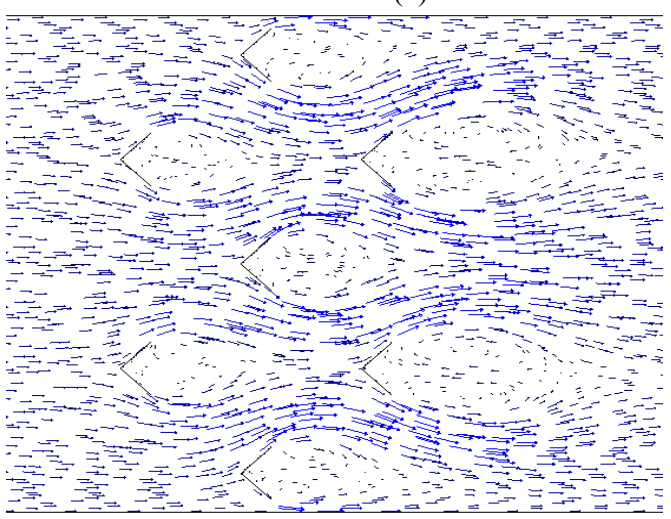

(d)

(a) $\mathrm{V}=1.0 \mathrm{H}$, (b) $\mathrm{V}=1.5 \mathrm{H}$, (c) $\mathrm{V}=2.0 \mathrm{H}$, (d) $\mathrm{V}=3.0 \mathrm{H}$

FIGURE 2. Velocity vectors of flow field in the turbulence coalescence section with different $\mathrm{V}$ values. 
At the inlet air velocity of $10 \mathrm{~m} / \mathrm{s}$, numerical simulation of the coagulation section of the turbulence coalescence were carried out separately with different angle diaphragms of $\mathrm{L}=1.0 \mathrm{~W}, \mathrm{~L}=2.0 \mathrm{~W}, \mathrm{~L}=3.0 \mathrm{~W}, \mathrm{~L}=5.0 \mathrm{~W}$. Fly ash particle size is $2.5 \mu \mathrm{m}$. The velocity vectors of flow field in the turbulence coalescence section with different $\mathrm{V}$ values is shown in Fig.3.

As can be seen from Fig.3, the turbulence appears around the angle diaphragms along the direction of the flow. The maximum velocity gradient exists in (a), but the pressure drop is also the largest. The velocity values tend to be uniformly distributed in (d). The effect of changing direction is best in (a). The horizontal spacing between two diaphragm groups is the smallest, as a result, the pressure drop of air flow through the device is also the largest. So, with the increase of $\mathrm{L}$, the effect of turbulence will be worse, but too small $\mathrm{L}$ value will cause excessive device pressure drop. $\mathrm{L}=2.0-3.0 \mathrm{~W}$ is an optimal choice.

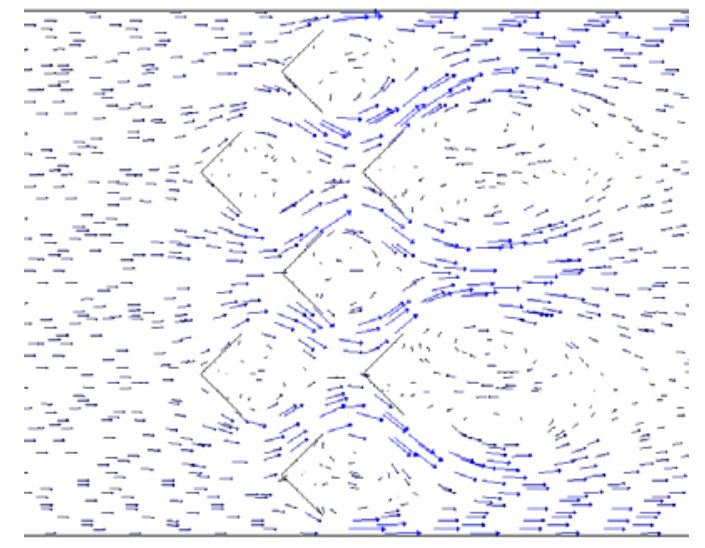

(a)

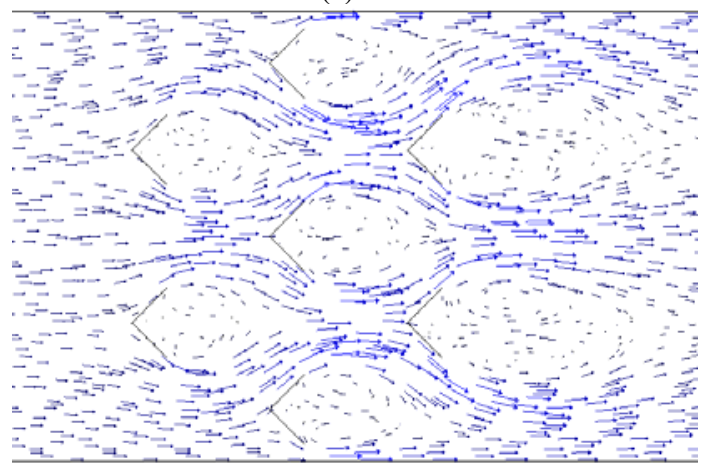

(c)

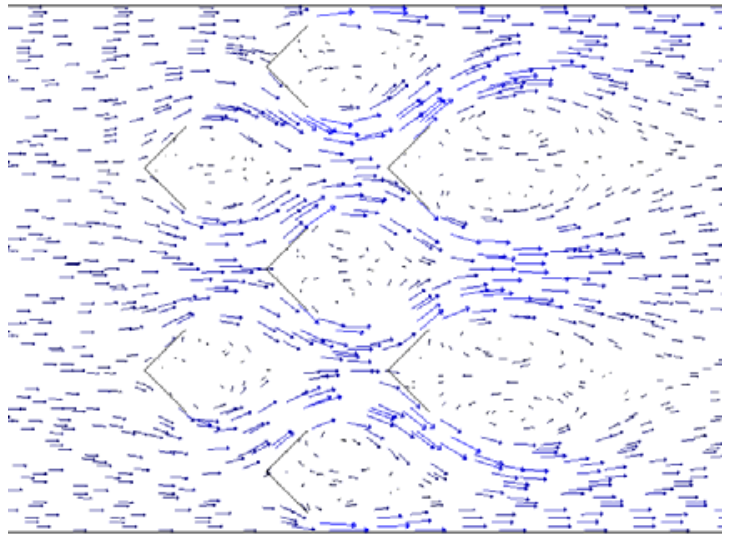

(b)

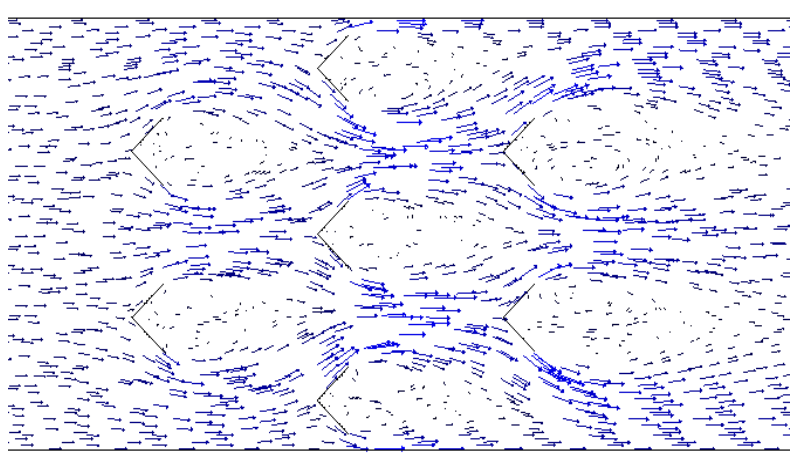

(d)

(a) $\mathrm{L}=1.0 \mathrm{~W}$, (b) $\mathrm{L}=2.0 \mathrm{~W}$, (c) $\mathrm{L}=3.0 \mathrm{~W}$, (d) $\mathrm{L}=5.0 \mathrm{~W}$

FIGURE 3. Velocity vectors of flow field in the turbulence coalescence aero with different $L$ values.

\section{SUMMARY}

The angle diaphragms were set up in fracture surface of the turbulence coalescence section of the coalescence device. Different velocity values and different velocity directions of partial flow in the flow field are generated, which could increase the probability of the collision of ultrafine particles and the particles aggregation. Considering the vortices and the pressure drop, "1.5-2.0 times the height of the diaphragm for vertical spacing between two diaphragms" and "2.0-3.0 times the length of the diaphragm for horizontal spacing between two diaphragm groups" are thought of optimal design. 


\section{REFERENCES}

1. Watanabe, T, Toehikubo, F., Koizurni, Y., et al. Submicron Particle agglomeration by an electrostatic agglomerate. [J] Journal of Electrostatics. Vol. 34 (1995) No. 4, p. 367-383.

2. ZHANG Gangue, LIU Jianzhong, ZHOU Jehu, et al. Simulation and analysis of collision efficiency in acoustic agglomeration [J], CIESC Journal, Vol. 60 (2009) No. 1, p. 42-46.

3. Han Xiao LIU, Zheng LIU.et, Multiple Vortex Body Vortex Numerical Simulation [J], Advanced Materials Research, Vol. 32 (2011) No. 14, p. 1755-1758.

4. Han Gang. Superfine powder coagulation device vortex generating section segment layout optimization research (Master Degree, North China Electric Power University, China,2013) p. 1-10. 